\title{
Bacterial tail anchors can target to the mitochondrial outer membrane
}

\author{
Güleycan Lutfullahoğlu-Bal ${ }^{1}$, Abdurrahman Keskin ${ }^{1,3}$, Ayşe Bengisu Seferoğlu ${ }^{1}$ and Cory D. Dunn ${ }^{1,2^{*}}$ (D)
}

\begin{abstract}
Background: During the generation and evolution of the eukaryotic cell, a proteobacterial endosymbiont was re-fashioned into the mitochondrion, an organelle that appears to have been present in the ancestor of all present-day eukaryotes. Mitochondria harbor proteomes derived from coding information located both inside and outside the organelle, and the rate-limiting step toward the formation of eukaryotic cells may have been development of an import apparatus allowing protein entry to mitochondria. Currently, a widely conserved translocon allows proteins to pass from the cytosol into mitochondria, but how proteins encoded outside of mitochondria were first directed to these organelles at the dawn of eukaryogenesis is not clear. Because several proteins targeted by a carboxyl-terminal tail anchor (TA) appear to have the ability to insert spontaneously into the mitochondrial outer membrane (OM), it is possible that self-inserting, tail-anchored polypeptides obtained from bacteria might have formed the first gate allowing proteins to access mitochondria from the cytosol.

Results: Here, we tested whether bacterial TAs are capable of targeting to mitochondria. In a survey of proteins encoded by the proteobacterium Escherichia coli, predicted TA sequences were directed to specific subcellular locations within the yeast Saccharomyces cerevisiae. Importantly, TAs obtained from DUF883 family members ElaB and YqjD were abundantly localized to and inserted at the mitochondrial OM.

Conclusions: Our results support the notion that eukaryotic cells are able to utilize membrane-targeting signals present in bacterial proteins obtained by lateral gene transfer, and our findings make plausible a model in which mitochondrial protein translocation was first driven by tail-anchored proteins.
\end{abstract}

Reviewers: This article was reviewed by Michael Ryan and Thomas Simmen.

Keywords: Protein targeting, Membrane insertion, Eukaryogenesis, Organelle biogenesis, Endosymbiosis

\section{Background}

During the incorporation of an $\alpha$-proteobacterial endosymbiont within the eukaryotic cell, genes transferred to the (proto)nucleus were re-targeted to mitochondria, allowing these organelles to remain the location of crucial cellular processes [1-3]. In addition, other polypeptides that evolved within the eukaryotic lineage or that were acquired through lateral gene transfer from other organisms were directed to mitochondria [4-6]. Across eukaryotes, the $\beta$-barrel Tom 40 protein forms a pore by which proteins pass through the OM [7-9].

\footnotetext{
* Correspondence: cory.dunn@helsinki.fi

${ }^{1}$ Department of Molecular Biology and Genetics, Koç University, 34450 Sarıyer, İstanbul, Turkey

${ }^{2}$ Institute of Biotechnology, Helsinki Institute of Life Science, University of Helsinki, P.O. Box 56, 00014 Helsinki, Finland

Full list of author information is available at the end of the article
}

However, the Tom40 polypeptide seems to require already existing TOM complexes for mitochondrial insertion $[10,11]$, giving rise to a "chicken or the egg" dilemma when considering how the TOM complex may have evolved.

Several narratives might be proposed for how mitochondria first evolved the ability to transport proteins from the cytosol. In one scenario, an early translocation pore that was self-inserting at the mitochondrial surface might have allowed mitochondria to begin to import proteins, permitting the subsequent evolution of the translocon found in eukaryotes today [12]. Current evidence suggests that the self-insertion of tail-anchored proteins at the mitochondrial OM is possible [13-15], and some tail-anchored pro-apoptotic proteins appear to have the ability to generate membrane pores at 
mitochondria [16, 17], making tenable such a scenario for the evolution of mitochondrial protein import. At the inception of mitochondria, such tail-anchored proteins would likely have been derived from prokaryotes, particularly if mitochondria were required for the generation of the stereotypical compartmentalized structure of eukaryotes.

We focused our attention upon a single aspect of this hypothesis: can TAs obtained from bacterial proteins be inserted into the mitochondrial $\mathrm{OM}$ when expressed within a eukaryotic cell? Indeed, our results demonstrate insertion and function at the mitochondrial OM for predicted TAs encoded by the proteobacterium E. coli, and we describe the relevance of our findings to the concept of lateral gene transfer during eukaryogenesis.

\section{Results}

\section{Bacterial tail anchors can localize to mitochondria}

To test whether predicted bacterial TAs might have the capacity to be inserted at the mitochondrial $\mathrm{OM}$, we identified 12 E. coli proteins predicted to harbor a solitary $\alpha$-helical transmembrane (TM) domain at the polypeptide carboxyl-terminus (Additional file 1: Figure S1), then fused mCherry to the amino-terminus of these TAs and examined their location in S. cerevisiae cells by fluorescence microscopy. mCherry-ElaB(TA) (Fig. 1a) and mCherry-YqjD(TA) (Fig. 1b) were readily detectable at mitochondria, as reported by co-localization with superfolder GFP (sfGFP) [18] fused to the TA of the $S$. cerevisiae Fis1 polypeptide, a protein playing a role in yeast mitochondrial division. A small fraction of mCherry$\mathrm{ElaB}(\mathrm{TA})$ and mCherry-YqjD(TA) could also be detected at the endoplasmic reticulum (ER) (Additional file 2: Figure S2). ElaB and YqjD are members of the DUF883 family of proteins. Little is known about the function of DUF883 family members, but YqjD may recruit ribosomes to the E. coli plasma membrane during stationary phase [19].

Due to dual localization of mCherry-ElaB(TA) and mCherry-YqjD(TA), we investigated whether the targeting of these proteins might be influenced by ER-mitochondria encounter structures (ERMES). However, mCherry$\mathrm{ElaB}(\mathrm{TA})$ and mCherry-YqjD(TA) were not limited to ERMES, as defined by Mdm34p-containing puncta [20] (Additional file 3: Figure S3), and disruption of ERMES by deletion of Mdm34p did not affect distribution of these fusion proteins to the swollen mitochondria resulting from Mdm34p removal [21, 22] or in their limited localization to ER (Additional file 4: Figure S4).

Although negligible fluorescent signal was detectable by microscopy or flow cytometry (C. Dunn, unpublished results), mCherry-TcdA(TA) could also be visualized at mitochondria (Additional file 5: Figure S5A). TcdA (also called CsdL) catalyzes the modification of E. coli tRNAs [23]. Other predicted TAs derived from the E. coli

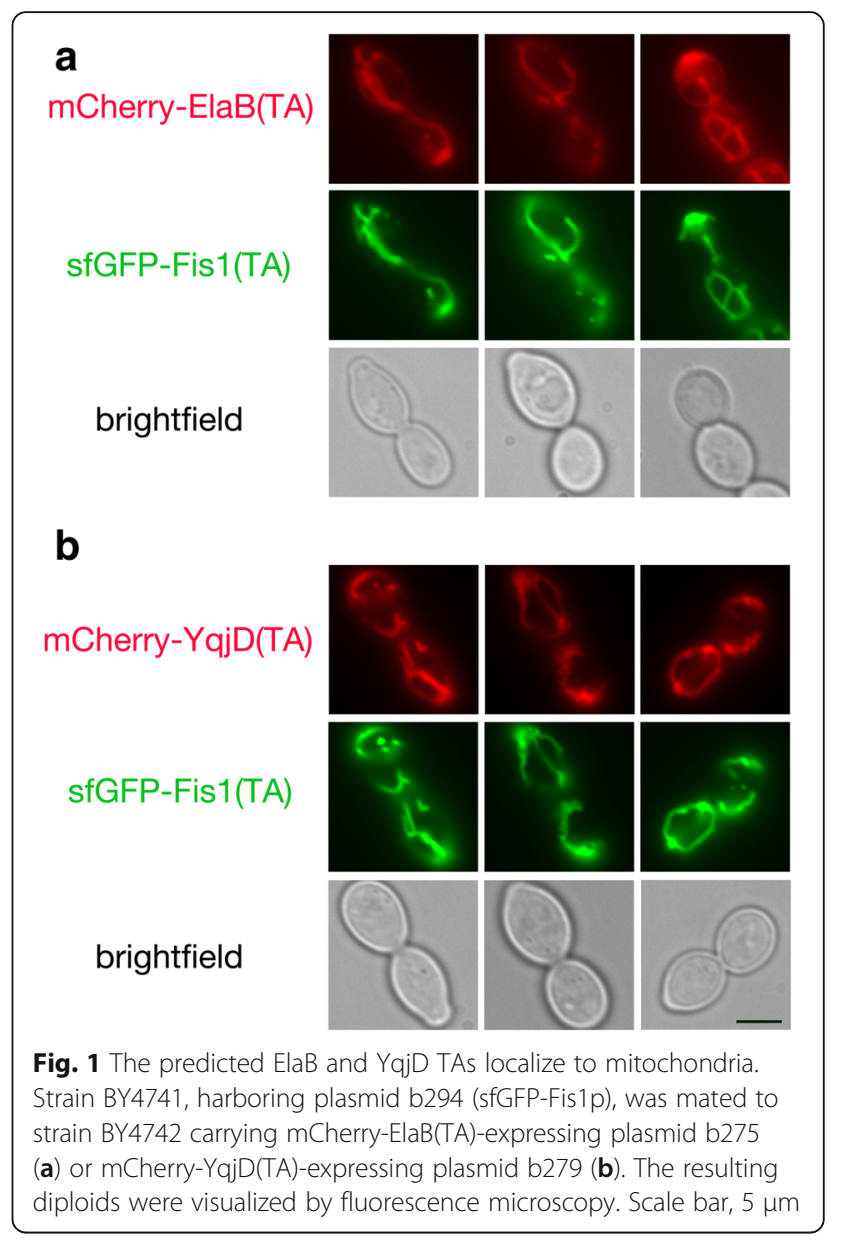

proteins Flk, YgiM, RfaJ, DjlB, FdnH, NrfF, and YmiA appeared to allow at least partial localization of mCherry to various locations associated with the endomembrane system (Additional file 6: Figure S6). However, no convincing localization to mitochondria was apparent after fusing any of these TAs to mCherry. The mCherryYhdV(TA) fusion protein appeared to be distributed throughout cytosol and nucleus, indicating failure to target efficiently to any membrane. mCherry-YgaM(TA) was not detectable, suggesting its degradation.

\section{Bacterial tail anchors can insert into membranes in a eukaryotic cell}

Previously, we developed an assay in which membrane insertion of proteins might be examined by a proliferationbased assay [24]. In brief, the Gal4 transcription factor is linked to a protein of interest that is thought to be inserted at a membrane outside of the nucleus. Failure of this fusion protein to insert at its target membrane can allow the Gal4-linked fusion protein to access the nucleus and activate Gal4-responsive promoters that drive proliferation under selective conditions. As previously demonstrated [24], while a membrane-sequestered Gal4-sfGFP-Fis1 
fusion protein did not lead to a proliferation defect on non-selective medium (SC-Trp), cells carrying this construct could not survive on medium requiring activation of a Gal4p-driven HIS3 gene (SMM-His +20 mM 3-AT) (Fig. 2). Deletion of the Fis1p TA, or the presence of a A144D charge substitution within the Fis1p TA, led to a failure of membrane insertion at mitochondria, translocation to the nucleus, and Gal4-dependent proliferation on selective medium. When the TA of Fis1p, a domain whose sole purpose is to allow this protein's insertion at the mitochondrial OM $[24,25]$, was replaced with the TA of either ElaB or YqjD, cells were unable to proliferate on medium selective for histidine synthesis, consistent with $\mathrm{ElaB}$ and YqjD TA insertion at the mitochondrial OM.

\section{Bacterial tail anchors can function at the mitochondrial outer membrane}

As these findings suggested that the ElaB and YqjD TAs may be competent for mitochondrial insertion, we tested whether these TAs can functionally replace the membranebound TA of Fis1p, thereby allowing Fis1p to promote mitochondrial division. Because Fis1p is required for mitochondrial fission in S. cerevisiae, mutants lacking this protein manifest a highly interconnected network of mitochondria due to unbalanced mitochondrial fusion [26-28]. As expected, expression of wild-type Fis1p restored normal mitochondrial distribution in this genetic background, while Fis1p prevented from mitochondrial insertion by a A144D substitution within the Fis1p TA [24] could not restore normal mitochondrial morphology (Fig. 3a and b). Strikingly, replacement of the Fis1p TA with the ElaB or the YqjD TA within the context of full length Fis1p polypeptide could successfully promote mitochondrial division and restore normal mitochondrial shape and distribution. A control TA obtained from the E. coli YgiM protein, which is not trafficked to mitochondria, could not support Fis1p activity. In addition, a Fis1-TcdA(TA) protein could not functionally replace the Fis1p TA in this microcopybased assay (Additional file 5: Figure S5B), suggesting insufficient expression, poor mitochondrial insertion, or meager functionality.

We then sought further evidence for functional insertion of the ElaB and YqjD TAs at the mitochondrial OM using an assay based upon cell proliferation [24]. Continued mitochondrial division after removal of a mitochondrial fusogen, such as Fzolp, can lead to mitochondrial fragmentation, loss of functional mitochondrial DNA (mtDNA), and a corresponding abrogation of respiratory competence [29-32]. This phenotype can be used as a basis to test Fis1p functionality [24]. As previously reported, expression of wild-type Fis1p in a fzo1 $1 \Delta$ fis $1 \Delta$ genetic background led to an inability to proliferate on non-fermentable medium, while expression of the poorly inserted Fis1(A144D) variant did not prompt mtDNA loss (Fig. 3c). The ElaB and YqjD TAs fused to the cytosolic domain of Fis1p allowed sufficient fission activity to prompt mitochondrial genome loss from the same genetic background, indicating successful ElaB TA and YqjD TA insertion at the mitochondrial OM. Even the Fis1TcdA(TA) protein provoked mtDNA loss in fzold fis1 $\Delta$ cells (Additional file 5: Figure S5C), suggesting some minimal level of OM insertion, while the YgiM TA again appeared unable to recruit Fis1p to mitochondria (Fig. 3c). Together, our results demonstrate insertion of the bacterial ElaB and YqjD TAs at the mitochondrial surface of a eukaryotic cell.

\section{Discussion}

Our findings, in which several predicted TAs obtained from E. coli can target to and function at the mitochondrial

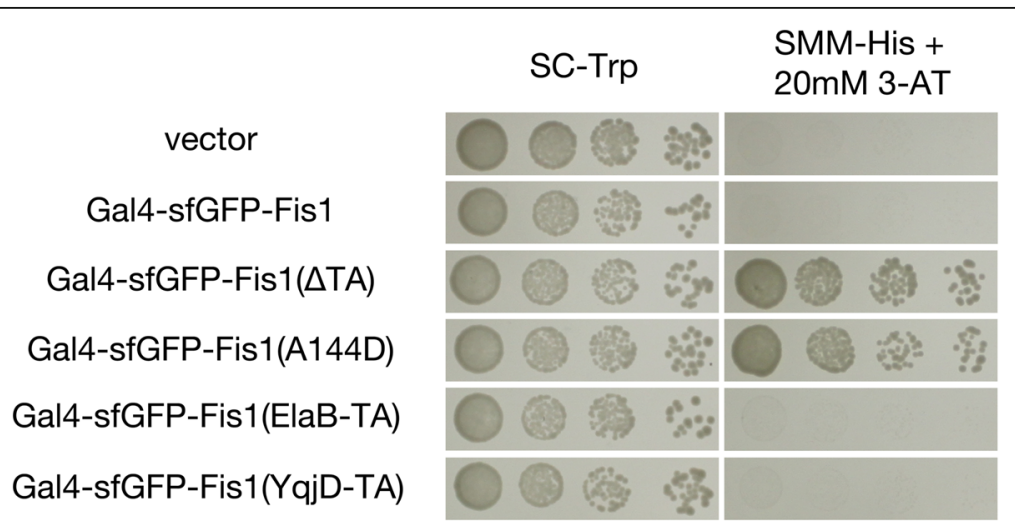

Fig. 2 A proliferation-based assay suggests that the ElaB and YqjD TAs are membrane inserted. Strain MaV203, containing a Gal4-driven HIS3 gene, was transformed with plasmids expressing Gal4-sfGFP-Fis1p (b100), a variant lacking the Fis1p TA (b101), a mutant containing the A144D charge substitution in its TA (b180), or the Gal4-sfGFP-Fis1p construct with the Fis1p TA replaced with that of either ElaB (b313) or YqjD (b314). MaV203 was also transformed with empty vector pKS1. Transformants were cultured in SC-Trp medium, then, following serial dilution, spotted to SC-Trp or SMM-His $+20 \mathrm{mM}$ 3-AT and incubated for $2 \mathrm{~d}$ 
a

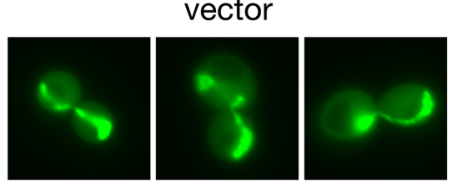

Fis1(A144D)p

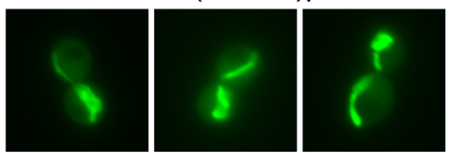

Fis1-YqjD(TA)p
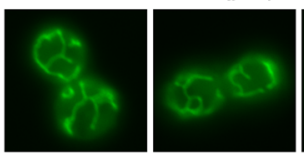

b

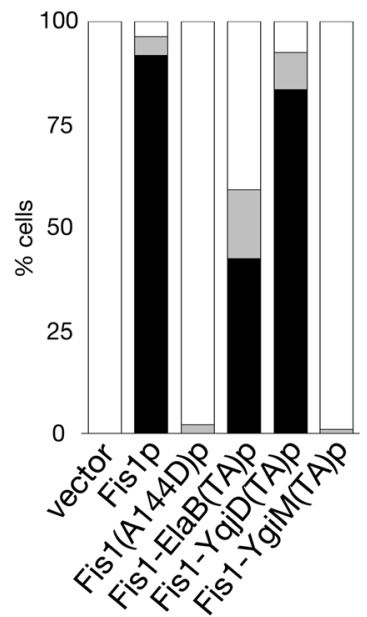

Fis1p

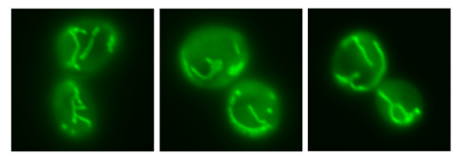

Fis1-ElaB(TA)p

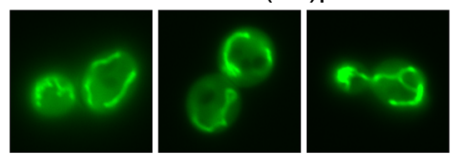

Fis1-YgiM(TA)p

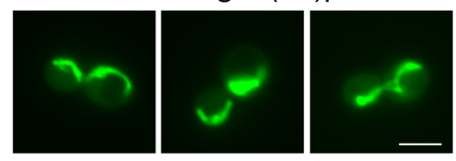

- Fis $1 p$

+ Fis $1 p$

counterselection counterselection

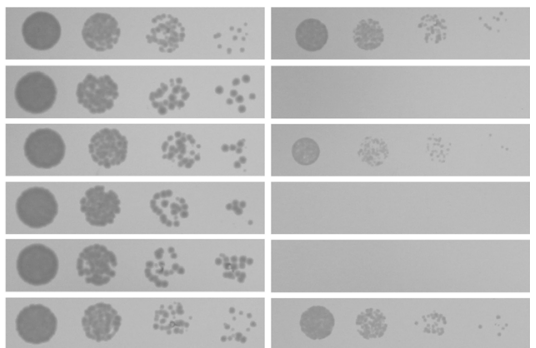

Fig. 3 Mitochondria-localized bacterial TAs can functionally replace the TA of Fis1p. a The ElaB and YqjD TAs can replace the Fis1p TA in promotion of normal mitochondrial morphology. fis $1 \triangle$ strain CDD741, expressing mitochondria-targeted GFP from plasmid pHS12, was transformed with empty vector pRS313 or plasmids expressing wild-type Fis1p (b239), Fis1 (A144D)p (b244), or Fis1p with its own TA replaced by that of ElaB (b317), YqjD (b318), or YgiM (b316). Cells were examined by fluorescence microscopy. Scale bar, $5 \mu \mathrm{m}$. b Quantification of mitochondrial morphology of the transformants from (a) was performed blind to genotype. White bar represents cells with fully networked mitochondria, grey bar represents cells with mitochondria not fully networked, but networked to a greater extent than wild-type cells, and black bar represents cells with normal mitochondrial morphology. Quantification was repeated three times ( $n>200$ per genotype), and a representative experiment is shown. c Genetic assessment of Fis $1 p$ variant functionality. Strain CDD688 was transformed with the plasmids used in (a) and proliferation was assessed without selection against Fis1 $p$ activity (YPALac medium for $2 \mathrm{~d}$ ) or following counter-selection for cells carrying functional Fis1p (SLac-His + CHX medium for $4 \mathrm{~d}$ )

OM of S. cerevisiae, make plausible a scenario in which tail-anchored bacterial proteins contributed to the formation of the earliest mitochondrial translocon. The structural characteristics of the TAs of ElaB and YqjD, a helical TM domain rich in glycines followed by a positively charged patch ending in di-arginine (Additional file 1: Figure S1), are evocative of the Fis1p TA, suggesting a similar, potentially spontaneous mechanism for insertion at mitochondria, although unassisted insertion of the ElaB and YqjD TAs at the mitochondrial surface has yet to be demonstrated. Notably, several conserved members of the current TOM complex are also tail-anchored [33], raising the possibility that at least some of these proteins could be "hold-overs" from an early, self-inserting mitochondrial translocon, although we note that these subunits cannot currently self-insert at mitochondria.

Could the DUF883 family of proteins have contributed to an ancestral mitochondrial OM translocon? While YqjD has been reported to recruit ribosomes to the $E$. coli inner membrane during stationary phase [19], a role in line with promotion of co-translational protein import into mitochondria [34, 35], the DUF883 family is not 
readily identified among eukaryotic genomes. One might expect, however, that once a more proficient TOM complex centered around the Tom 40 pore evolved, a previous translocon would have been lost, or even selected against if it were to interfere with more rapid protein import through an improved OM translocation machinery. Moreover, an inordinate focus on DUF883 family members when seeking components of the earliest mitochondrial translocon may not be warranted in any case, since the structural characteristics likely required for TA insertion at mitochondria might be easily generated from random open reading frame fragments containing a transmembrane domain. Analogously, random peptides from bacteria are readily able to act as amino-terminal mitochondrial targeting sequences [36-38]. If TAs are easily evolved and might recruit other functional domains to the mitochondrial surface, then identifying orthologs of initial tail-anchored translocon components from existing prokaryotic sequences might be difficult, since an untold number of TAs might be predicted among putative open-reading frames. Supporting the idea that mitochondrial TAs might be generated from sequences not actually functioning in membrane targeting within their native bacterial environment, we demonstrated limited mitochondrial targeting and partial functionality of the computationally predicted TcdA TA in yeast, even though TcdA is unlikely to be membraneinserted in E. coli [39].

If conversion of endosymbiont to mitochondrion were the rare and essential event required for generation of eukaryotes, and if insertion of bacteria-derived, tailanchored proteins at the $\mathrm{OM}$ to form an ancestral translocon were necessary for this conversion, then the question of how hospitable an environment the early mitochondrial OM might have been for bacteria-derived TAs comes to the fore [12]. Indeed, the membrane into which tail-anchored proteins are inserted can be at least partially determined by their lipid environment [13], and lipids utilized by many characterized archaea are fundamentally different in structure from bacterial and eukaryotic lipids [40]. However, recent evidence indicates that archaeal clades potentially related to the last eukaryotic common ancestor might have been characterized by membranes more similar to those of bacteria than of those membranes more typically found in archaea [41]. This finding raises the possibility that the proto-eukaryote's specific cohort of lipids was crucial to the ability to form complexes of bacteria-derived tailanchored proteins at the mitochondrial OM that might have allowed full integration of mitochondria within the ancestral eukaryote.

Finally, we have not examined in detail the trafficking of $E$. coli TAs that appeared to localize to the endomembrane system during our initial survey. However, the diverse organellar locations to which these TAs were localized supports previous data indicating that eukaryotes may derive organelle targeting information from newly acquired prokaryotic proteins or protein fragments, perhaps even from amino acid sequences previously unselected for targeting proficiency [36-38, 42, 43]. Lateral gene transfer promotes the evolution of novel functions in prokaryotes [44] and was certainly present in the form of endosymbiotic gene transfer during early eukaryogenesis. Indeed, proficiency in making use of cryptic or explicit targeting information in order to direct newly acquired, nucleus-encoded proteins to the distinct subcellular locations where they might be best utilized might have provided a significant selective advantage to the early eukaryote. Such a scenario may be particularly relevant if some amount of cellular compartmentalization already existed in a pre-eukaryotic host cell before conversion of pre-mitochondrial endosymbiont to organelle $[45,46]$.

\section{Conclusions}

We have demonstrated that TAs from bacteria can localize to and insert within the mitochondrial OM. Our results make plausible the suggestion that tail-anchored proteins acquired by bacteria could have formed an initial translocon at the mitochondrial outer membrane, and our findings indicate that certain membrane-bound proteins acquired by horizontal gene transfer could have easily found their way to diverse locations within eukaryotic cells at which they might provide a selective advantage. Further efforts will be necessary to determine whether self-inserting proteins or peptides may have generated the initial mitochondrial translocon.

\section{Methods}

\section{Yeast strains, plasmids, and culture conditions}

Culture conditions are as described in [24], and all experiments have been carried out at $30{ }^{\circ} \mathrm{C}$. Strains, plasmids, and oligonucleotides used in this study are found in Additional file 7.

\section{Selection of $E$. coli tail anchors subject to investigation}

FASTA sequences from the $E$. coli proteome were retrieved from UniProt [47] and analyzed using the TMHMM 2.0 server [48]. Polypeptides with a single predicted TM domain, harboring 15 or less amino acids carboxyl-terminal to the TM domain, and containing more than 30 amino acids amino-terminal to the TM domain were selected for further analysis.

\section{Microscopy}

Microscopy was performed on logarithmic phase cultures as in [24], with exposure times determined automatically. mCherry fusions are driven by the $A D H 1$ 
promoter and universally contain Fis1p amino acids 119-128 (not necessary or sufficient for mitochondrial targeting) linking mCherry to each TA. The brightness of all images of mCherry expression was adjusted in Adobe Photoshop CS5 (Adobe, San Jose, California) to an equivalent extent. Microscopic assessment of Fis1p variant functionality was performed as described in [24], and scoring of mitochondrial morphology was performed blind to genotype.

\section{Proliferation-based assessment of Fis1p insertion and functionality}

Genetic tests of Fis1p insertion and functionality were performed as in [24].

\section{Reviewers' comments}

\section{Reviewer 1: Michael T. Ryan, Monash University} General comments

In this manuscript, Bal and colleagues address elements of the endosymbiotic hypothesis notably whether tailanchored proteins from bacteria can directly insert into mitochondria and therefore have the potential to have formed the first components of the mitochondrial protein import machinery. While the translocation channel is a beta barrel and likely came from bacteria, the evolutionary origin of single transmembrane receptor proteins is not well established. Here the authors definitively show that a subset of tail anchored bacterial proteins can indeed target mitochondria when expressed in yeast cells. The work is very clear and the manuscript is exceedingly well written. I think this is an interesting and important piece of work that merits publication. I have no issues to report.

\section{Specific comments}

I have no issues with this manuscript it is extremely well written and well presented.

Authors' Response: We thank Dr. Ryan for his encouraging comments regarding our manuscript.

\section{Reviewer 2: Thomas Simmen, University of Alberta General comments}

$\mathrm{Bal}$ et al. present a thoughtprovoking study that investigates an intriguing question: can today's bacterial tailanchored proteins provide mitochondrial targeting information within a eukaryotic cell. The article warrants interest by a larger audience, but for the moment, some shortcomings must be addressed to make the manuscript more succinct and convincing. More specifically, some of the data are of rather subpar quality and there are some questions about the mtDNA depletion assay, which would lead some readers to dismiss the study. Given the originality of the topic, this should provide some solid insight into this attractive hypothesis.

\section{Specific comments}

This is a thoughtprovoking study that investigates an intriguing question: can today's bacterial tailanchored proteins provide mitochondrial targeting information within a eukaryotic cell. The article should generate interest by a larger audience, but for the moment, some shortcomings must be addressed to make the manuscript more succinct and convincing. The proposed improvements aim to make this a stronger paper.

Specific Point 1 . The microscopic technique used at the moment is unclear; the images appear a little blurred to draw the rather precise conclusions that the authors propose. For the localization studies that the paper advertises, confocal or deconvolution microscopy must be used.

Authors' Response 1: It is typical to detect some outof-focus light from spherical yeast cells when using epifluorescence light microscopy. Moreover, our experience with standard confocal microscopy of yeast is that image quality is generally not improved, due to reduced signal intensity and diminished ability to visualize mitochondrial morphology throughout the cell. To summarize, we feel that co-localization of mCherry-ElaB(TA) and mCherryYqjD(TA) with mitochondria is well documented here, with little to be gained by use of alternative microscopy approaches.

Incidentally, we noted upon upload of our files for this revision that the resolution is much reduced in the PDF generated for review. We can directly provide the 600dpi files upon request before publication, if the reviewer wishes.

Specific Point 2. The authors state that some constructs localize to the ER, but that is not obvious. It might be interesting to pursue this question, since some mitochondrial TA proteins might transit to mitochondria on ERmitochondria contact sites (see also below). Due to the potential relevance of this question, some colocalization should be done.

Authors' Response 2: Only a minor fraction of $m$ Cherry-ElaB(TA) and mCherry-YqjD(TA) constructs localize to ER. However, these proteins are quite clearly detectable at this subcellular location, as defined by use of Sec63p-GFP as an ER marker, in several of the representative images collected.

We agree that it is worthwhile to examine potential localization of these tail anchors with respect to ERmitochondria contact sides. Toward this goal, we compared the localization of mCherry-ElaB(TA) and mCherry$Y q j D(T A)$ to that of an ER-mitochondria encounter structure (ERMES) component, Mdm34p, fused to superfolder GFP. However, we found no evidence of concentration of the ElaB and YqjD TAs at ERMES. These new results are found in Additional file 3: Figure S3.

In addition, we disrupted ERMES by removal of Mdm34p, but we detected no change to the localization 
of $m$ Cherry-ElaB(TA) or $m C h e r r y-Y q j D(T A)$ : the majority of each protein was targeted to mitochondria and a minority of each protein was found at the ER. These data are provided in Additional file 4: Figure S4.

Specific Point 3. mCherry TcdA(TA) is claimed to partially localize to mitochondria. I cannot see this with the current image quality. This should be improved and potentially complemented with fractionation studies.

Authors' Response 3: As described in the text, the signal of $m$ Cherry-TcdA(TA) is quite weak, suggesting that the protein may be unstable. Much of the mCherry-TcdA(TA) that can be detected appears generally distributed throughout the cytosol of the cell. This might be expected for a sequence that, contrary to our computational prediction, does not actually function as a TA in bacteria [39]. However, some small fraction of the mCherry-TcdA(TA) construct is evidently directed to mitochondria, as indicated by co-localization with sfGFP-Fis1(TA). Most importantly, mtDNA loss occurring specifically when Fis1$T c d A(T A) p$ is expressed in cells lacking both Fzolp and wild-type Fis1p (Additional file 5: Figure S5C) demonstrates by genetic means that the TcdA TA can be inserted at the mitochondrial outer membrane.

Specific Point 4. On page 5 at the beginning of the bottom chapter, the authors claim that their results suggest that ElaB and YqiD mediate insertion into mitochondrial membranes, but at this point of the text, they have only presented evidence for membrane, not mitochondrial insertion.

Authors' Response 4: We thank the reviewer for this correction, and we have changed the relevant text from, "As these findings indicated..." to "As these findings suggested..."

Specific Point 5. On Fig. 3a, i do not see any evidence that ElaB TA rescues targeting of Fis1 and, hence, mitochondrial fission.

Authors' Response 5: We maintain that our images show an overt rescue of mitochondrial morphology for cells expressing Fis1-ElaB(TA)p when compared to cells expressing non-functional Fis1(A144D)p or Fis1-YgiM(TA)p, or not expressing any Fis1p variant at all (vector control). Indeed, mitochondrial morphology was also quantified blind to genotype (Fig. 3b), further validating our conclusion that Fis1-ElaB(TA)p is functional.

Specific Point 6. Since TcdA also leads to loss of mtDNA in S3C, but does not show mitochondrial targeting, the authors should provide an assay, which can detect also small amounts of mitochondrial targeting, ideally fractionation.

Authors' Response 6: Some mCherry-TcdA(TA) is localized to mitochondria, as described above (Specific Point 3) and seen in (what is now) Additional file 5: Figure S5A.

Moreover, our genetic assay argues for some minimal amount of mitochondrial insertion, since Fis1-TcdA(TA) $p$ can provoke mtDNA loss in cells deleted of both Fis1p and Fzo1p. We expect that our genetic assay is a more sensitive readout of Fis1p function when compared to a microscopybased assay. If sufficient fission takes place at any time during the generation of a cell pedigree, mtDNA is permanently lost. In contrast, a microscopy-based assay may not easily capture instances at which Fis $1 p$ construct activity is sufficiently high to promote rescue of mitochondrial morphology that would be readily discernable by fluorescence microscopy. To summarize, our localization of $m$ Cherry-TcdA(TA) to mitochondria, combined with the results of our genetic assay, demonstrate that a small population of proteins terminating in a predicted TcdA TA can insert into the mitochondrial outer membrane.

We would like to thank Dr. Simmen for his helpful comments regarding our manuscript.

\section{Additional files}

Additional file 1: Figure S1. A list of predicted TAs examined in this study. The UniProt accession number and names of selected proteins are provided, along with the sequences of the predicted TAs. The single predicted TM domain is denoted by a purple line. Charged amino acids are also indicated. For purposes of sequence comparison, the relevant portion of the S. cerevisiae Fis1p TA is also shown. (TIFF $526 \mathrm{~kb}$ )

Additional file 2: Figure S2. The predicted ElaB and YqjD TAs can also be visualized at the endoplasmic reticulum. Cells harboring (A) plasmid b275 [mCherry-ElaB(TA)] or (B) plasmid b279 [mCherry-YqjD(TA)] were analyzed as in Fig. 1, except BY4741 was transformed with plasmid pJK59, expressing Sec63p-GFP, before mating. In addition, cells expressing mCherry-Fis1(TA) from plasmid b109 are shown for comparison (C). Scale bar, $5 \mu \mathrm{m}$. (TIFF 5526 kb)

Additional file 3: Figure S3. The predicted ElaB and YqjD TAs are not specifically localized to ERMES. Strain CDD1210, expressing Mdm34p-sfGFP, was transformed with (A) plasmid b275 [mCherry-ElaB(TA)] or (B) plasmid b279 [mCherry-YqjD(TA)] and visualized by fluorescence microscopy. Scale bar, 5 нm. (TIFF 3859 kb)

Additional file 4: Figure S4. Disruption of ERMES does not affect trafficking of ElaB and YqjD TAs to mitochondria and ER. $m d m 34 \triangle$ strain CDD1209 was transformed with plasmid b275 [mCherry-ElaB(TA)] (A and $B)$ or plasmid b279 [mCherry-YqjD(TA)] (C and D). The mitochondrial matrix was labelled using pHS1 [Cox4p(1-21)-GFP] (A and C), and the ER membrane was labelled using pJK59 (Sec63p-GFP) (B and D). Cells were visualized by fluorescence microscopy. Scale bar, 5 um. (TIFF 6631 kb)

Additional file 5: Figure S5. The predicted TcdA TA allows minimal localization to, and function at, the mitochondrial outer membrane. (A) The predicted TcdA TA can be visualized at mitochondria. Strain BY4741, harboring plasmid b294 (sfGFP-Fis1p), was mated to strain BY4742 carrying mCherry-TcdA(TA)-expressing plasmid b281 and the resulting diploids were imaged by fluorscence microscopy. Scale bar, $5 \mu \mathrm{m}$. (B) Fis1 p with its own TA replaced by the predicted TcdA TA cannot provide detectable Fis1p activity as assessed by visualizing mitochondrial morphology. fis $1 \triangle$ strain CDD741, expressing mitochondria-targeted GFP from plasmid pHS12, was transformed with empty vector pRS313 or plasmids expressing wild-type Fis1p (b239), Fis1(A144D)p (b244), or Fis1-TcdA(TA)p (b319) and mitochondrial morphology was examined. (C) Fis1-TcdA(TA)p can allow mitochondrial division. Strain CDD688 was transformed with the plasmids used in (B) or a plasmid expressing Fis1-YgiM(TA)p (b316) and examined as in Fig. 3c, except that culture on medium counter-selective for Fis $1 p$ activity was carried out for $5 \mathrm{~d}$. (TIFF $5312 \mathrm{~kb}$ )

Additional file 6: Figure S6. Not all predicted E. coli TAs are localized to mitochondria in S. cerevisiae. Strain CDD961 was transformed with 
plasmids expressing (A) mCherry-Flk(TA) (b273), (B) mCherry-YhdV(TA) (b277) (C) mCherry-RfaJ(RA) (b278), (D) mCherry-DjB(TA) (b280), (E) mCherny-FdnH(TA) (b331), (F) mCherry-NrfF(TA) (b332), or (G) mCherry-YmiA(TA) (b333) and examined by fluorescence microscopy. (H) Strain BY4741, carrying plasmid b311 expressing sfGFP fused to the enhanced PTS1 sequence [49], was mated to strain BY4742, containing the mCherry-YgiM(TA)-expressing plasmid b274, and the resulting diploids were imaged. (TIFF $9892 \mathrm{~kb}$ )

Additional file 7: Strains, plasmids, and oligonucleotides used during this study [24, 32, 50-56]. (XLS 49 kb)

\section{Abbreviations}

ER: Endoplasmic reticulum; ERMES: ER-mitochondria encounter structure; mtDNA: Mitochondrial DNA; OM: Outer membrane; sfGFP: Superfolder GFP; TA: Tail anchor; TM: Transmembrane

\section{Acknowledgements}

We thank Thomas Richards and Jeremy Wideman for comments on this manuscript.

\section{Funding}

This work was supported by a European Research Council Starting Grant (637649-RevMito) to C.D.D., by a Turkish Academy of Sciences Outstanding Young Scientist Award (TÜBA-GEBIP) to C.D.D., and by Koç University. These funding bodies had no role in the design of the study, data collection, data analysis, data interpretation, or manuscript preparation.

\section{Availability of data and materials}

All data generated or analyzed during this study are included in this published article and its Additional files 1, 2, 3, 4, 5, 6 and 7.

\section{Authors' contributions}

CDD designed the study, wrote the manuscript, and performed experiments. GLB, AK, and ABS performed experiments, generated reagents, and provided manuscript critiques. All authors read and approved the final manuscript.

\section{Ethics approval and consent to participate}

None required.

\section{Consent for publication}

None required.

\section{Competing interests}

The authors declare that they have no competing interests.

\section{Publisher's Note}

Springer Nature remains neutral with regard to jurisdictional claims in published maps and institutional affiliations.

\section{Author details}

${ }^{1}$ Department of Molecular Biology and Genetics, Koç University, 34450 Sarıyer, İstanbul, Turkey. ${ }^{2}$ Institute of Biotechnology, Helsinki Institute of Life Science, University of Helsinki, P.O. Box 56, 00014 Helsinki, Finland. ${ }^{3}$ Present Address: Department of Biological Sciences, Columbia University, New York, NY 10027, USA.

Received: 23 March 2017 Accepted: 10 July 2017

Published online: 24 July 2017

\section{References}

1. Booth A, Doolittle WF. Eukaryogenesis, how special really? Proc Natl Acad Sci U S A. 2015;112:10278-85.

2. Hewitt V, Alcock F, Lithgow T. Minor modifications and major adaptations: the evolution of molecular machines driving mitochondrial protein import Biochim Biophys Acta. 2011;1808:947-54.

3. Gray MW. Mosaic nature of the mitochondrial proteome: implications for the origin and evolution of mitochondria. Proc Natl Acad Sci U S A. 2015; 112:10133-8.

4. De Duve C. The origin of eukaryotes: a reappraisal. Nat Rev Genet. 2007;8:395-403.
5. Kurland CG, Collins LJ, Penny D. Genomics and the irreducible nature of eukaryote cells. Science. 2006;312:1011-4.

6. Gabaldón T, Huynen MA. Reconstruction of the proto-mitochondrial metabolism. Science. 2003:301:609.

7. Mani J, Meisinger C, Schneider A. Peeping at TOMs-diverse entry gates to mitochondria provide insights into the evolution of eukaryotes. Mol Biol Evol. 2016;33:337-51.

8. Shiota T, Imai K, Qiu J, Hewitt VL, Tan K, Shen H-H, et al. Molecular architecture of the active mitochondrial protein gate. Science. 2015;349:1544-8.

9. Hill K, Model K, Ryan MT, Dietmeier K, Martin F, Wagner R, et al. Tom40 forms the hydrophilic channel of the mitochondrial import pore for preproteins. Nature. 1998;395:516-21.

10. Rapaport D, Neupert W. Biogenesis of Tom40, core component of the TOM complex of mitochondria. J Cell Biol. 1999;146:321-31.

11. Model K, Meisinger C, Prinz T, Wiedemann N, Truscott KN, Pfanner N, et al, Multistep assembly of the protein import channel of the mitochondrial outer membrane. Nat Struct Biol. 2001;8:361-70.

12. Renthal R. Helix insertion into bilayers and the evolution of membrane proteins. Cell Mol Life Sci. 2009:67:1077-88.

13. Krumpe K, Frumkin I, Herzig Y, Rimon N, Özbalci C, Brügger B, et al. Ergosterol content specifies targeting of tail-anchored proteins to mitochondrial outer membranes. Mol Biol Cell. 2012;23:3927-35.

14. Kemper C, Habib SJ, Engl G, Heckmeyer P, Dimmer KS, Rapaport D. Integration of tail-anchored proteins into the mitochondrial outer membrane does not require any known import components. J Cell Sci. 2008;121:1990-8.

15. Setoguchi K, Otera H, Mihara K. Cytosolic factor- and TOM-independent import of C-tail-anchored mitochondrial outer membrane proteins. EMBO J. 2006:25:5635-47.

16. Große L, Wurm CA, Brüser C, Neumann D, Jans DC, Jakobs S. Bax assembles into large ring-like structures remodeling the mitochondrial outer membrane in apoptosis. EMBO J. 2016;35:402-13.

17. Salvador-Gallego R, Mund M, Cosentino K, Schneider J, Unsay J, Schraermeyer $U$, et al. Bax assembly into rings and arcs in apoptotic mitochondria is linked to membrane pores. EMBO J. 2016:35:389-401.

18. Pédelacq J-D, Cabantous S, Tran T, Terwilliger TC, Waldo GS. Engineering and characterization of a superfolder green fluorescent protein. Nat Biotechnol. 2005;24:79-88.

19. Yoshida H, Maki Y, Furuike S, Sakai A, Ueta M, Wada A. YqjD is an inner membrane protein associated with stationary-phase ribosomes in Escherichia coli. J Bacteriol. 2012;194:4178-83.

20. Kornmann B, Currie E, Collins SR, Schuldiner M, Nunnari J, Weissman JS, et al. An ER-mitochondria tethering complex revealed by a synthetic biology screen. Science. 2009;325:477-81.

21. Dimmer KS, Fritz S, Fuchs F, Messerschmitt M, Weinbach N, Neupert W, et al. Genetic basis of mitochondrial function and morphology in Saccharomyces cerevisiae. Mol Biol Cell. 2002;13:847-53.

22. Youngman MJ, Hobbs AEA, Burgess SM, Srinivasan M, Jensen RE. Mmm2p, a mitochondrial outer membrane protein required for yeast mitochondrial shape and maintenance of mtDNA nucleoids. J Cell Biol. 2004;164:677-88.

23. Miyauchi K, Kimura S, Suzuki T. A cyclic form of N6-threonylcarbamoyladenosine as a widely distributed tRNA hypermodification. Nat Chem Biol. 2013;9:105-11.

24. Keskin A, Akdoğan E, Dunn CD. Evidence for Amino Acid Snorkeling from a High-Resolution, In Vivo Analysis of Fis1 Tail-Anchor Insertion at the Mitochondrial Outer Membrane. Genetics. 2017;691-705.

25. Habib SJ, Vasiljev A, Neupert W, Rapaport D. Multiple functions of tailanchor domains of mitochondrial outer membrane proteins. FEBS Lett. 2003:555:511-5.

26. Mozdy AD, McCaffery JM, Shaw JM. Dnm1p GTPase-mediated mitochondrial fission is a multi-step process requiring the novel integral membrane component Fis1p. J Cell Biol. 2000;151:367-80.

27. Fekkes P, Shepard KA, Yaffe MP. Gag3p, an outer membrane protein required for fission of mitochondrial tubules. J Cell Biol. 2000:151:333-40.

28. Tieu Q, Nunnari J. Mdv1p is a WD repeat protein that interacts with the dynamin-related GTPase, Dnm1p, to trigger mitochondrial division. J Cell Biol. 2000;151:353-66.

29. Hermann GJ, Thatcher JW, Mills JP, Hales KG, Fuller MT, Nunnari J, et al. Mitochondrial fusion in yeast requires the transmembrane GTPase Fzo1p. J Cell Biol. 1998:143:359-73.

30. Rapaport D, Brunner M, Neupert W, Westermann B. Fzo1p is a mitochondrial outer membrane protein essential for the biogenesis of functional mitochondria in Saccharomyces cerevisiae. J Biol Chem. 1998;273:20150-5. 
31. Bleazard W, McCaffery JM, King EJ, Bale S, Mozdy A, Tieu Q, et al. The dynamin-related GTPase Dnm1 regulates mitochondrial fission in yeast. Nat Cell Biol. 1999;1:298-304.

32. Sesaki $H$, Jensen RE. Division versus fusion: Dnm1p and Fzo1p antagonistically regulate mitochondrial shape. J Cell Biol. 1999;147:699-706.

33. Burri L, Lithgow T. A complete set of SNAREs in yeast. Traffic. 2003;5:45-52.

34. Verner K. Co-translational protein import into mitochondria: an alternative view. Trends Biochem Sci. 1993;18:366-71.

35. Williams CC, Jan CH, Weissman JS. Targeting and plasticity of mitochondrial proteins revealed by proximity-specific ribosome profiling. Science. 2014; 346:748-51.

36. Baker A, Schatz G. Sequences from a prokaryotic genome or the mouse dihydrofolate reductase gene can restore the import of a truncated precursor protein into yeast mitochondria. Proc Natl Acad Sci U S A. 1987;84:3117-21.

37. Lemire BD, Fankhauser C, Baker A, Schatz G. The mitochondrial targeting function of randomly generated peptide sequences correlates with predicted helical amphiphilicity. J Biol Chem. 1989;264:20206-15.

38. Lucattini R, Likic VA, Lithgow T. Bacterial proteins predisposed for targeting to mitochondria. Mol Biol Evol. 2004;21:652-8.

39. Kim S, Lee H, Park S. The structure of Escherichia Coli TcdA (also known as CsdL) reveals a novel topology and provides insight into the tRNA binding surface required for N6-Threonylcarbamoyladenosine Dehydratase activity. J Mol Biol. 2015;427:3074-85.

40. Lombard J, López-García P, Moreira D. The early evolution of lipid membranes and the three domains of life. Nat Rev Microbiol. 2012;10:507-15.

41. Villanueva L, Schouten S, Damsté JSS. Phylogenomic analysis of lipid biosynthetic genes of Archaea shed light on the "lipid divide". Environ Microbiol. 2016:19:54-69.

42. Hall J, Hazlewood GP, Surani MA, Hirst BH, Gilbert HJ. Eukaryotic and prokaryotic signal peptides direct secretion of a bacterial endoglucanase by mammalian cells. J Biol Chem. 1990;265:19996-9.

43. Walther DM, Papic D, Bos MP, Tommassen J, Rapaport D. Signals in bacterial beta-barrel proteins are functional in eukaryotic cells for targeting to and assembly in mitochondria. Proc Natl Acad Sci U S A. 2009:106:2531-6.

44. Treangen TJ, EPC R. Horizontal transfer, not duplication, drives the expansion of protein families in prokaryotes. PLoS Genet. 2011;7:e1001284-12.

45. Pittis $\mathrm{AA}$, Gabaldón T. Late acquisition of mitochondria by a host with chimaeric prokaryotic ancestry. Nature. 2016;531:101-4.

46. Zaremba-Niedzwiedzka K, Caceres EF, Saw JH, Bäckström D, Juzokaite L, Vancaester $\mathrm{E}$, et al. Asgard archaea illuminate the origin of eukaryotic cellular complexity. Nature. 2017;541:353-8.

47. The UniProt Consortium. UniProt: the universal protein knowledgebase. Nucleic Acids Res. 2017;45:D158-69.

48. Krogh A, Larsson B, Heijne von G, Sonnhammer ELL. Predicting transmembrane protein topology with a hidden markov model: application to complete genomes. J Mol Biol. 2001;305:567-80.

49. DeLoache WC, Russ ZN, Dueber JE. Towards repurposing the yeast peroxisome for compartmentalizing heterologous metabolic pathways. Nat Commun. 2016:7:1-11.

50. Sikorski RS, Hieter P. A system of shuttle vectors and yeast host strains designed for efficient manipulation of DNA in Saccharomyces cerevisiae. Genetics. 1989;122:19-27.

51. Prinz WA, Grzyb L, Veenhuis M, Kahana JA, Silver PA, Rapoport TA. Mutants affecting the structure of the cortical endoplasmic reticulum in Saccharomyces cerevisiae. J Cell Biol. 2000;150:461-74.

52. Ryan KR, Leung RS, Jensen RE. Characterization of the mitochondrial inner membrane translocase complex: the Tim23p hydrophobic domain interacts with Tim17p but not with other Tim23p molecules. Mol Cell Biol. 1998;18:178-87.

53. Taxis C, Knop M. System of centromeric, episomal, and integrative vectors based on drug resistance markers for Saccharomyces cerevisiae. BioTechniques. 2006;40(1):73-8.

54. Lee S, Lim WA, Thorn KS. Improved blue, green, and red fluorescent protein tagging vectors for S. cerevisiae. PLoS One. 2013;8:e67902.

55. Garipler G, Mutlu N, Lack NA, Dunn CD. Deletion of conserved protein phosphatases reverses defects associated with mitochondrial DNA damage in Saccharomyces cerevisiae. Proc Natl Acad Sci U S A. 2014;111:1473-8.

56. Mutlu N, Garipler G, Akdoğan E, Dunn CD. Activation of the pleiotropic drug resistance pathway can promote mitochondrial DNA retention by fusion-defective mitochondria in Saccharomyces cerevisiae. G3 (Bethesda). 2014:4:1247-58.

\section{Submit your next manuscript to BioMed Central and we will help you at every step:}

- We accept pre-submission inquiries

- Our selector tool helps you to find the most relevant journal

- We provide round the clock customer support

- Convenient online submission

- Thorough peer review

- Inclusion in PubMed and all major indexing services

- Maximum visibility for your research

Submit your manuscript at www.biomedcentral.com/submit
Biomed Central 